\title{
ON ANOMALIES IN INTERACTING SPIN-1 FIELD THEORIES
}

\author{
A, AINSAAR, M. KOIV. ANOMAALIATEST INTERAGEERIVATE, SPINNIGA 1 VĂLJADE TEOO- \\ RIATES \\ А. АННСААР, М. КЫПВ. ОБ АНОМАЛИЯХ В ТЕОРИЯХ ВЗАИМОДЕПСТВУЮЩИХ ПОЛЕП \\ Со спином 1
}

Anomalies of the theories for higher spins (both in quantized and classical versions) are well known $\left.{ }^{1}\right]$. Some of these difficulties arise already in the case of such a "low" spin value as one. Except normal interacting spin-1 field theories there are those being both noncausal and noncovariant $\left[{ }^{1,2}\right]$ or only noncovariant $\left[{ }^{3}\right]$.

We have considered $\left.{ }^{4}\right]$ another pathological aspect of interacting spin-1 field theories which become evident when expanding the S-matrix into Feynman graphs.

Let us take a general form of one-boson interaction Lagrangian

$$
L_{\mathrm{int}}=-j_{\mu}^{*} \Phi_{\mu}-\frac{1}{2} j_{v, \mu}^{*}\left(\partial_{\mu} \Phi_{v}-\partial_{v} \Phi_{\mu}\right)+\text { h.c. },
$$

where $\Phi_{\mu}$ is a spin-1 boson field operator in the Heisenberg representation, $j^{*}$ contains other fields with spin values $0,1 / 2$ and massless 1 . The corresponding interaction Hamiltonian for constructing the S-matrix in the approach of Umezawa and Takahashi $\left[{ }^{5}\right]$ takes the form

$$
H_{\mathrm{int}}=j_{\mu}^{*} \Phi_{\mu}+\frac{1}{2} j_{v, \mu}^{*}\left(\partial_{\mu} \Phi_{v}-\partial_{v} \Phi_{\mu}\right)+\frac{1}{2 m^{2}}\left|j_{4}\right|^{2}+\frac{1}{2} j_{\mu, 4}^{*} j_{\mu, 4}+\text { h.c. },
$$

where $\Phi$ is in the interaction representation. The terms without $\Phi$-field indicate the presence of direct coupling of the other fields, which was not evidently included in (1). tion

Somewhat different peculiarities arise in the case of two-boson interac-

$$
L_{\mathrm{int}}=Q_{A}^{*} M_{A B} Q_{B} ; \quad Q_{A}=\left(\Phi_{\mu}, \partial_{v} \Phi_{\rho}-\partial_{\rho} \Phi_{v}\right),
$$

which in general leads to infinite series in corresponding interaction Hamiltonian

$$
H_{\mathrm{int}}=-Q_{A}^{*} Q_{B} \sum_{n=1}^{\infty}\left(F_{n} M^{n}\right)_{A B}
$$


where the numbers $F_{n}$ can sometimes be more plainly expressed in terms of Kemmer-Duffin quantities. Here one can see* that a graph vertex can include an unlimited number of lines of the other fields in addition to one passing boson line.

Thus, the interactions can be divided into two classes depending on whether the abnormalities of S-matrix expansion do occur or not. The analysis can be easily performed by means of the results of $\left[{ }^{4}\right]$.

For the interactions considered in $\left[{ }^{3}\right]$ it can be concluded that

(i) the interaction $\Phi^{\mu} W_{\mu v} \Phi^{v}$ which is causal and covariant if $W_{\mu v}$ is antisymmetric and which violates both if $W_{\mu v}$ is symmetric, turns out to have finite expression of $H_{\text {int }}$ (4) in the antisymmetric case and infinite series in case $W_{\mu v}$ is symmetric,

(ii) the interaction $\Phi_{\lambda} \partial^{\lambda} F_{\mu v} \partial^{\mu} \Phi^{v}$, which violates both causality and covariance, is also abnormal in our point of view, i. e., it retains infinite series in (4),

(iii) the derivative one-boson tensor coupling with a spin- $\frac{1}{2}$ field $\bar{\psi} \sigma^{\mu v} \psi \partial_{\mu} \varphi_{v}$ known as causal but noncovariant, contains a self-coupling of the Dirac field,

(iv) every two-boson derivative coupling with a spin- $\frac{1}{2}$ field leads to infinite series in $H_{\text {int }}(4)$. These are known as noncovariant whereas only one of them (the vector one) is causal.

Thus, the S-matrix expansion anomalies seem to be in a certain correlation with non-covariance and, perhaps, in less immediate relations with noncausality.

* Both anomalies arise in spin-0 boson interactions as well.

\section{REFERENCES}

1. Velo, G., Z w a n zi g e r, D., Phys. Rev., 186, 1357 (1969); 188, 2218 (1969).

2. Jenkins, J. D., J. Phys., A6, 1935 (1973); A7, 1129 (1974).

3. B a b u, Joseph K., S a bi r, M., J. Phys., A9, 1951 (1976).

4. A in s a a r, A., Kồ i v, M., Preprint FAI-25, Tartu, 1973.

5. U m e z a w a, H., Quantum field theory, Amsterdam, 1956.

Note added in proof - After the submission of the present letter for publication the results of Guertin and Wilson [R. F. Guertin, T. L. Wilson, Phys. Rev. D15, 1518 (1977)] became known to us. They show that in the case of spin 0 every tensor coupling (and only tensor one) in the Kemmer-Duffin formalism leads to noncausality. It can be seen in $\left[{ }^{4}\right]$ that this is just the case where the expansion anomalies occur. 